\title{
Protein Nanocapsules Produced by Nanoprecipitation of Designed Suckerin-Silk Fusion Proteins
}

\author{
Ricardo Ramos, Kenrick Koh, Bartosz Gabryelczyk ${ }^{\dagger}$, Luxiao Chai, Kanagavel Deepankumar, Xibo Yan, François Ga- \\ nachaud, Ali Miserez, * Julien Bernard*
}

\begin{abstract}
Herein, we report on the precise design of a modular fusion protein amenable to the construction of nanocapsules by nanoprecipitation. The central squid suckerin-derived peptide block provides structural stability, whereas both termini from silk fibroins make the modular protein highly soluble at physiological $\mathrm{pH}$, a critical requirement for the nanoprecipitation process. With this molecular design, nanocapsules consisting of suckerin-silk fusion protein shells and hexadecane or miglyol cores with sizes in the range $190-250 \mathrm{~nm}$ are straightforwardly built.
\end{abstract}

Polymer nanocapsules are progressively emerging as promising candidates for drug delivery applications thanks to their potential to improve targeting, drug efficiency, reduced side effects, their aptitude to co-deliver multiple drugs or their ability to cross physiological barriers including the cell membrane. ${ }^{1,2}$ Owing to specific physico-chemical and biological properties including inherent biocompatibility, low immunogenicity, biodegradability, complete control of the (amino acid) sequence, or the suitability to diverse functionalization with bioactive domains, proteins are a particularly advantageous class of building blocks for the construction of nanocapsules. ${ }^{3,4}$ The most common proteinaceous nanocapsules for encapsulation of drugs are self-assembled protein nanocages, such as ferritin or vault nanostructures, which are made of protein subunits that self-assemble into nanoscaled cages with precise geometry. ${ }^{3}$ While this biological approach leads to ordered protein nanostructures with exquisite control over their geometry and near monodisperse size distributions, it is unfortunately limited to specific protein building blocks that selfassemble via precisely-defined protein-protein interactions of the subunits..$^{5}$ Alternatively, the construction of proteinaceous nanocapsules can be undertaken by means of chemical or physico-chemical approaches. The prevalent routes rely on multi-step template driven strategies such as layer-by-layer technique ${ }^{6}$, complex coacervation emulsification technique ${ }^{7}$, or interfacial reactions of proteins in water-in-oil miniemulsion systems. ${ }^{8,9}$

Nanoprecipitation is a simple and easy-to-use "bottom-up" strategy typically relying on the supersaturation of a hydrophobic solute (such as an oil, first dissolved in a water-miscible solvent) upon addition of water, and has lately emerged as a general and modular method to generate polymer nanocapsules with a precise control of the dimensions and the physico-chemical properties. The first step of nanoprecipitation is to establish phase diagrams and to identify the domains of interest, e.g. Ouzo and Surfactant-Free MicroEmulsion (SFME) regions. ${ }^{10}$ This information can then be used to establish conditions of solvent shifting so that the solute is spontaneously emulsified and forms submicronic templates, onto which hydrophilic polymer chains (initially dissolved in the aqueous phase) quantitatively precipitate to grow a robust polymer shell. Following this route, oil-filled nanocapsules have been constructed from a range of vinyl-based polymers ${ }^{11,12}$ and polysaccharides ${ }^{13}$. However, to the best of our knowledge, the preparation of protein-based nanocapsules by nanoprecipitation has not been reported yet.

A recently discovered family of structural proteins, suckerins, has shown promising potential for biomedical applications ${ }^{14,15}$ (including as therapeutics carriers). Suckerins are modular, block copolymerlike structural proteins that constitute the hard sucker ring teeth of squids and cuttlefish. ${ }^{16}$ They consist of 10-12 amino acid-long modules forming $\beta$-sheets that are interspersed by longer, mostly amorphous domains ${ }^{17,18}$ (although protein NMR experiments indicate that short peptides in these domains can also form $\beta$-sheet secondary structures ${ }^{19,20,21}$ ), resulting in mechanically-robust materials with good processability. ${ }^{22}$ Another important biochemical feature of suckerins is their high Histidine (His) amino acid content (ca. 10 mol.\%). Because of the pKa (ca. 6.0) of its imidazole sidechain, His is protonated under mild acidic conditions, which not only enhances the solubility of suckerins in water but also facilitates endosomal escape via the proton-sponge effect. ${ }^{14}$ While suckerins can be readily produced recombinantly in $E$. coli with full control of the amino acid sequence, ${ }^{23}$ they usually aggregate in the inclusion bodies during expression because of the relatively high content of hydrophobic residues, requiring subsequent treatment with high concentration urea in order to solubilize them. ${ }^{23}$ Moreover, although purified recombinant suckerins are soluble at acidic $\mathrm{pH}$, their solubility significantly decreases in aqueous buffers around $\mathrm{pH}$ 7. This $\mathrm{pH}$-dependent solubility is beneficial to fabricate films or other solid materials ${ }^{21,22}$ but may constitute a barrier for obtaining nanocapsules by nanoprecipitation. Indeed, the efficiency of the process is generally compromised when $\mathrm{pH}$ values are below 5 or above $10,{ }^{24}$ and self-assembly or aggregation of the proteins is not desirable before solvent shifting.

In this context, we aim herein at developing simple nanoprecipitation routes to nanocapsules involving suckerin-based recombinant proteins and water/acetone/oil ternary systems. Starting from previously reported recombinant proteins from suckerin-19, we then describe how engineering of fusion suckerin proteins with enhanced solubility in water can be achieved by fusing a silk-derived solubility tag to a central suckerin domain, thereby affording the production of proteinaceous materials with aqueous solution behavior at $\mathrm{pHs}$ compatible with a solvent shifting procedure (see Figure S1). We further establish these recombinant suckerin-silk fusion proteins as promising modality to produce protein-based functional nanocapsules through nanoprecipitation in a straightforward manner.

We first tested suckerin-19 in nanoprecipitation assays. The two amino acids with ionizable side-chains in suckerin-19 are tyrosine (Tyr) and His (ca. 15\% and 10\% of the protein composition) residues. ${ }^{19,20,21}$ These residues possess $\mathrm{R}$ groups with pKa's around 6 (imidazole of His) and 10 (hydroxyl of Tyr). The protein net charge is thus nil in the $\mathrm{pH}$ range between 6 and 10 so the chains tend to 
(a)
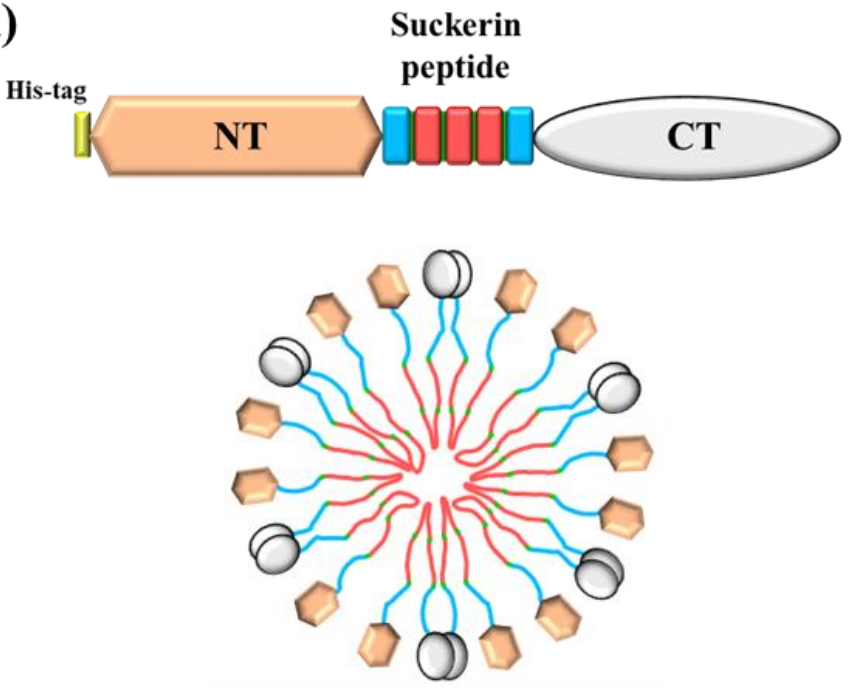

(b)

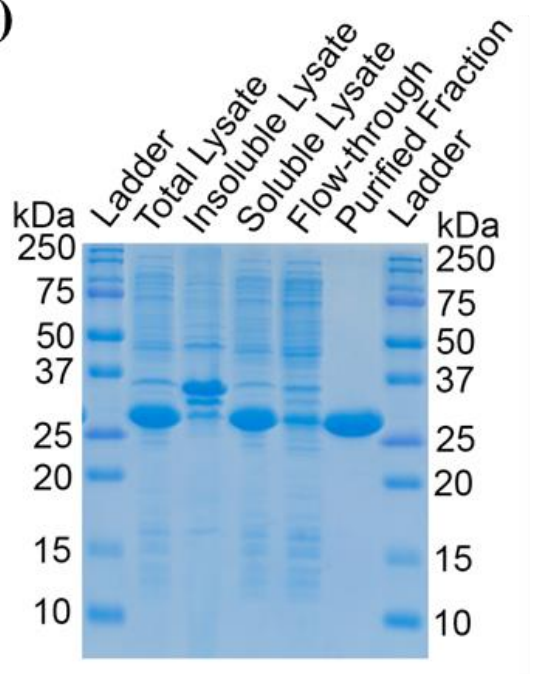

(e)

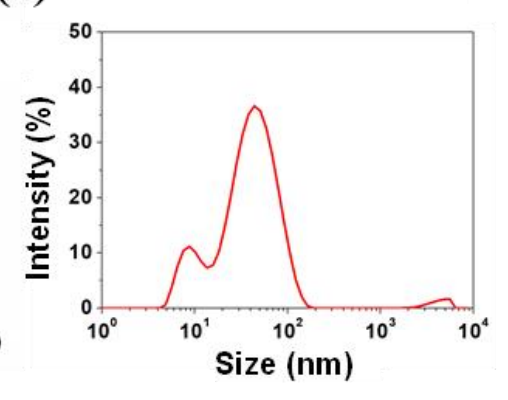

Figure 1. (a). (top) Modular representation of suckerin silk fusion protein (NT-SUP-CT). NT, suckerin peptides, and CT sequences definitions and sequences are given in Tables 1 and 2; (bottom) schematic representation of micellar-like soluble structures generated in water thanks to the hydrophilicity of N- and C-termini domains (adapted after Ref. 28). (b) SDS-PAGE of NT-SUP-CT fusion protein. Bio-Rad Precision Plus Protein Standards Dual Color was used as a reference ladder. (c) CD spectra of NT-SUP-CT at varying temperatures (blue: $20^{\circ} \mathrm{C}$, red: $40^{\circ} \mathrm{C}$, blacl: $60^{\circ} \mathrm{C}$ ). (d) Maldi-ToF spectrum of NT-SUP-CT fusion protein. The main $\mathrm{m} / z$ peak at $33 \mathrm{kDa}$ corresponds to the expected MW of NT-SUP-CT fusion protein. Peak at $16.5 \mathrm{kDa}$ corresponds to the doubly charged $(z=2)$ species. (e) DLS spectra of suckerin silk dissolved in a $20 \mathrm{mM}$ aqueous buffer at $\mathrm{pH} 7.4$ and a concentration of $3.5 \mathrm{mg} / \mathrm{ml}$.

aggregate (Figure S2). Since acidic pHs (at which suckerin 19 chains are closer to monomeric state, $\mathrm{pH}=3$ ) impair the nanoprecipitation process, we first intended to generate nanocapsules through solvent shifting at $\mathrm{pH} \sim 10$ (see DLS of aqueous protein solutions at $\mathrm{pH} 10$, Figure S3). Under such conditions, the solvent shifting procedure yielded ill-defined mixtures of protein capsules together with protein aggregates in the range of a few hundred nanometers as evidenced by TEM analyses (see Figure S4 in supporting information).

We then decided to design a fusion protein containing suckerin peptide domains and exhibiting water solubility in appropriate $\mathrm{pH}$ conditions for nanoprecipitation. In a recent study, Andersson et al. ${ }^{25}$ demonstrated that engineered spider silk proteins (fibroins) could be produced in E. coli in soluble form and at remarkably high yield (in the range of 200-300 mg/mL). Like suckerins, spidroins contain a long repetitive, hydrophobic central domain that forms strong $\beta$-sheet structures in silk fibres. This domain is flanked by nonrepetitive N-terminus (NT) and C terminus (CT) globular domains, whose roles are to maintain a high solubility of the spidroins in the silkforming gland of the spider and to trigger aggregation into $\beta$-sheet rich structures during silk drawing. ${ }^{26,27}$ Exploiting the NT domain of a spidroin from Euprosthenops australis (E. australis) and the CT domain of a spidroin from Araneus ventricosus (A. ventricosus), this group was able to produce proteins and peptides that are notoriously difficult to express in soluble form, including membrane proteins or amyloidogenic peptides. ${ }^{28,29}$ Based on such results, we hypothesized that these NT and CT tags could be fused to suckerins or suckerin peptides as a way to enhance their solubility near neutral $\mathrm{pH}$. We constructed a fusion protein consisting of central suckerin-derived peptide (SUP) domain flanked by NT and CT tags (NT-SUP-CT) (Figure 1a). ${ }^{30}$ The SUP domain contains three copies of the peptide module PAATAVSHTHHA that was previously shown to form cross- $\beta$ functional amyloids ${ }^{30}$, separated by di-Glycine linkers. Furthermore, to bring chain flexibility to the construct, the $\beta$-sheet forming modules were flanked by two copies of the Glycine (Gly) and Tyrosine (Tyr)-rich amorphous peptide GLGGYGGLYGGY, also found abundantly in suckerins. ${ }^{17}$

Our results show that NT-SUP-CT fusion protein can be recombinantly expressed in E. coli in soluble form, with a high yield of ca. $100 \mathrm{mg} / \mathrm{L}$ of recombinant bacterial culture. NT-SUP-CT can be readily purified using nickel affinity chromatography in aqueous buffer without the need of using denaturing agents, and can be dissolved at neutral $\mathrm{pH}(>150 \mathrm{mg} / \mathrm{mL})$, a solution behavior that could not be achieved with wild-type suckerins or SUP lacking the NT and CT domains. In comparison, previous production yields were in the range of $30 \mathrm{mg} / \mathrm{L}$ of cell culture, highlighting the efficiency of NT and CT tags not only to enable soluble expression but also to enhance expression yield. As shown by SDS-PAGE (Figure 1c) and MALDIToF (Figure 1d) analysis, high purity NT-SUP-CT was obtained after dialysis with the expected molecular weight (MW) of $33.1 \mathrm{kDa}$. Circular dichroism (CD) spectra (Figure 1d) showed the presence of two minima at 208 and $220 \mathrm{~nm}$ at room temperature, which is the signature of $\alpha$-helices present in the NT and CT tags. At increasing 
Table 1. Protein construct, sequence design, and its source. Protein accession numbers are provided when available.

\begin{tabular}{|c|c|c|c|c|c|}
\hline \multirow{2}{*}{$\begin{array}{l}\text { Construct } \\
\text { Name }\end{array}$} & \multicolumn{4}{|c|}{ Amino Acid Sequence } & \multirow{2}{*}{ Source } \\
\hline & His tag & NT region & Center Region & CT region & \\
\hline NT-SUP-CT & МННННННН & $\begin{array}{l}\text { E. australis MaSp1 NT region } \\
\text { (UniProtKB accession number } \\
\text { Q05H60) }\end{array}$ & $\begin{array}{l}\text { D. gigas suckerin peptide. } \\
3 \text { repeats of Ala- and His- } \\
\text { rich domains flanked with } \\
\text { Gly-rich peptides }\end{array}$ & $\begin{array}{l}\text { A. ventricocus MiSp } \\
\text { CT region } \\
\text { (UniProtKB acces- } \\
\text { sion number } \\
\text { K4MTL7) }\end{array}$ & GenScript \\
\hline
\end{tabular}

Table 2. Amino acid sequence of specific regions/peptides used in construct design. For D. gigas suckerin module, peptides highlighted in blue are Gly-rich amorphous domains; Ala-rich and His-rich domains forming $\beta$-sheets are highlighted in red and proline residues in green. Gly di-peptides (highlighted in yellow) were added between the $\beta$-sheets peptides as linkers.

\begin{tabular}{|l|l|}
\hline Sequence Name & Amino Acid Sequence \\
\hline $\begin{array}{l}\text { E. australis MaSp1 } \\
\text { NT region }\end{array}$ & $\begin{array}{l}\text { MSHTTPWTNPGLAENFMNSFMQGLSSMPGFTASQLDDMSTIAQSMVQSIQSLAAQGRTSPNKLQALNM } \\
\text { AFASSMAEIAASEEGGGSLSTKTSSIASAMSNAFLQTTGVVNQPFINEITQLVSMFAQAGMNDVSA }\end{array}$ \\
\hline $\begin{array}{l}\text { A. ventricocus MiSp } \\
\text { CT region }\end{array}$ & $\begin{array}{l}\text { VTSGGYGYGTSAAAGAGVAAGSYAGAVNRLSSAEAASRVSSNIAAIASGGASALPSVISNIYSGVVASG } \\
\text { VSSNEALIQALLELLSALVHVLSSASIGNVSSVGVDSTLNVVQDSVGQYVG }\end{array}$ \\
\hline D. gigas suckerin peptides & $\begin{array}{l}\text { GLGGYGGLYGGYPAATAVSHTTHHAPGGPAATAVSHTTHHAPGGPAATAVSHTTHHAPGLGGYGGLY } \\
\text { GGY }\end{array}$ \\
\hline
\end{tabular}
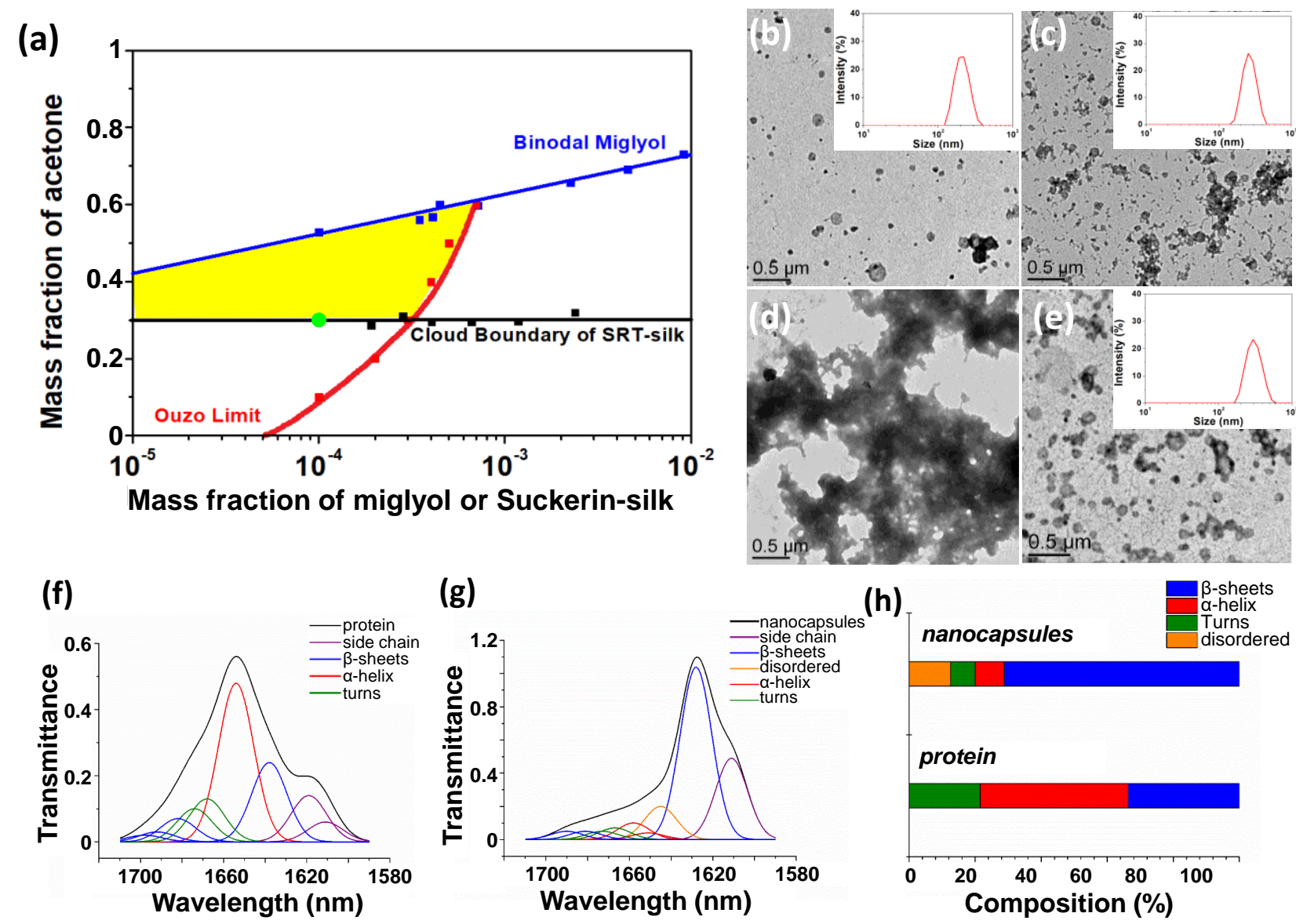

Figure 2. Fabrication of nanocapsules with shells made of NT-SUP-CT. (a) Overlapped phase diagrams of the systems miglyol/acetone/water and suckerin silk/acetone/20 mM tris aqueous buffer. Domain of coprecipitation colored in yellow. (b to e) TEM images and DLS spectra showing the effect of protein and crosslinker contents on nanocapsule fabrication (full TEM photos available in Fig. S8). All nanocapsules were prepared at 30/70 acetone/aqueous buffer ratio and $10^{-4}$ mass fraction of miglyol. (b) $10^{-5}$ mass fraction of NT-SUP-CT and $6.5 \times 10^{-6}$ mass fraction of IPDI. (c) $5 \times 10^{-5}$ mass fraction of NT-SUP-CT and $6.5 \times 10^{-6}$ mass fraction of IPDI. (d) $10^{-4}$ mass fraction of suckerin-silk and $6.5 \times 10^{-6}$ mass fraction of IPDI. (e) $10^{-5}$ mass fractions of NT-SUP-CT and $1.6 \times 10^{-6}$ mass fraction of IPDI. Amide I region of the Fourier-transform infrared spectroscopy (FTIR) spectrum of (f) suckerin-silk dissolved in the aqueous buffer and (g) protein nanocapsules dispersed in the aqueous buffer. (h) Overview of the secondary structure shift of the suckerin-silk protein before and after nanocapsule formulation, showing increase in $\beta$-sheets content after nanocapsules formation 
Table 3. Sizes and size distributions of the miglyol/NT-SUP-CT nanocapsules measured by DLS and TEM.

\begin{tabular}{|c|c|c|c|c|c|c|}
\hline Sample & Suckerin-silk mass fraction & IPDI mass fraction & $\overline{\boldsymbol{D}}_{\boldsymbol{z}, \boldsymbol{T E M}}((\mathrm{nm})$ & $\overline{\boldsymbol{D}}_{\boldsymbol{w}, \boldsymbol{T E M} \boldsymbol{M}} / \overline{\boldsymbol{D}}_{\boldsymbol{n}, \boldsymbol{T E M}}$ & $\overline{\boldsymbol{D}}_{\boldsymbol{z}, \boldsymbol{D L S} \boldsymbol{(}}(\mathrm{nm})$ & PDI \\
\hline B & $10^{-5}$ & $6.5 \times 10^{-6}$ & 191 & 1.34 & 200 & 0.10 \\
\hline C & $5 \times 10^{-5}$ & $3.3 \times 10^{-6}$ & 187 & 1.35 & 250 & 0.20 \\
\hline D & $10^{-4}$ & $6.5 \times 10^{-5}$ & n.d $^{1}$ & -n.d $^{1}$ & n.d. $^{1}$ & n.d. $^{1}$ \\
\hline E & $10^{-5}$ & $1.6 \times 10^{-6}$ & 220 & 1.35 & 300 & 0.20 \\
\hline
\end{tabular}

${ }^{1}$ Not determined. Formation of large micrometer-scale aggregates

temperatures, the spectra displayed an upwards shift in the 205-230 $\mathrm{nm}$ range, and a smoothening of the two minima at 208 and $220 \mathrm{~nm}$ into a broader minimum at $215 \mathrm{~nm}$. These features are characteristic of an $\alpha$-helix to $\beta$-sheet transition of the tag domains upon heating.

We next investigated the behaviour of the suckerin fusion protein in aqueous $20 \mathrm{mM}$ Tris buffer solution at $\mathrm{pH} 7.4$ at a concentration of $3.5 \mathrm{mg} / \mathrm{ml}$ by DLS to confirm that NT-SUP-CT proteins are theoretically amenable to the construction of precisely defined nanocapsules by nanoprecipitation. DLS analysis of the aqueous protein solutions is given in Figure 1f and shows two peaks at $9 \mathrm{~nm}$ and $50 \mathrm{~nm}$ corresponding to free unimers and tiny micellar aggregates of NTSUP-CT. Importantly, the intensity ratio between the two peaks indicates that under such conditions, most of the proteins present themselves as unimers in the solution, making it ideal to obtain nanocapsules by solvent shifting. As a comparison, wild type suckerins are typically dissolved in an aqueous buffer containing 5\% (v/v) acetic acid (pH 3) and DLS analysis of the solution containing $1 \mathrm{mg} / \mathrm{ml}$ of suckerin-19 shows two peaks at 30 and $220 \mathrm{~nm}$ (data not shown).

The next step consisted in seeking the best nanoprecipitation compositions in phase diagrams. Previously, our group derived the acetone/water/miglyol phase diagram and identified the Ouzo domain. ${ }^{13}$ To find the conditions needed for nanocapsule fabrication, the cloud point boundary of NT-SUP-CT in acetone/aqueous buffer was added here. This limit corresponds to the solubility limit of the protein in the mixture.Its determination method consists in titrating various aqueous solutions of suckerin-silk protein with the organic solvent until the mixtures turns turbid (see experimental part for details). Overlapping the two phase diagrams (see Figure 2a) allows the identification of a solvent shifting domain where, ideally, protein chains and the oil co-precipitate to generate nanocapsules. ${ }^{13}$ The fact that NT-SUP-CT is initially dissolved in Tris buffer (of rather low ionic strength) is not expected to drastically change the boundaries determined here. Inspection of the diagram reveals that the acetone content must be between 30 and $50 \mathrm{wt} \%$ for an oil mass fraction of $10^{-4}$ to prepare nanocapsules inside the ouzo domain. To crosslink the nanocapsules shell, isophorone diisocyanate (IPDI) with a final mass fraction of a $6.5 \times 10^{-6}$ is also added to the organic phase. The diisocyanate is reactive towards $-\mathrm{NH}_{2}$ and $-\mathrm{OH}$ groups available in the protein forming urea and urethane linkages respectively, capable of crosslinking the shells.

At an acetone content of $\sim 30 \mathrm{wt} \%$, clean samples of nanocapsules (Figure $2 \mathrm{~b}$ ) with an average diameter $\bar{D}_{Z D L S}$ around $200 \mathrm{~nm}$ $\left(\bar{D}_{Z T E M} \sim 190 \mathrm{~nm}\right)$, a dispersity $\left(\bar{D}_{w} / \bar{D}_{n}\right)$ of 1.35 measured by TEM, and a PDI of 0.10 measured by DLS were obtained. Proteinaceous nanocapsules are in the same size range as some polysaccharidebased nanocapsules but slightly bigger than those typically obtained from glycopolymer chains and miglyol in the Ouzo domain (dimensions below $150 \mathrm{~nm}$ ). This probably stems from a less favorable stabilization of the oil droplets by the proteins, as suggested by the concomitant observation of few nanoparticles in TEM photos. It is also worth mentioning that contrary to polysaccharide-based nanocapsules, for which $\bar{D}_{Z D L S}$ and $\bar{D}_{Z T E M}$ are usually quite different, ${ }^{13}$ these values are very close for NT-SUP-CT-based NCs (200 vs $190 \mathrm{~nm}$ ), indicating a poor swelling of the suckerin silk membrane in solution.
Note that the interval conditions to prepare nanocapsules is narrow. At an acetone content of $50 \mathrm{wt} \%$, large dark domains were spotted revealing inefficient encapsulation of the oil (see Figure S5a), and the sample could not be analyzed by DLS due to the presence of large protein aggregates (not seen with the naked eye). Decreasing the acetone weight fraction to $40 \%$ (Figure S5b) resulted in protein nanoparticles together with nanocapsules. The observed nanocapsules possess a mean diameter of $110 \mathrm{~nm}$ as measured by TEM, but DLS analysis did not provide insights into the size distribution due to the presence of large aggregates. Higher acetone content than $30 \%$ thus seems to promote a harsh competition between self-aggregation of protein chains and precipitation at the surface of oil droplets Protein concentration plays an essential role on the control of nanocapsule formation. Figure 2 shows three samples prepared at an acetone content of $30 \mathrm{wt} \%$, a miglyol mass fraction of $10^{-4}$ and a protein mass fraction of $10^{-5}$ (Figure $2 \mathrm{~b}$ ), $5 \times 10^{-5}$ (Figure $2 \mathrm{c}$ ) and $10^{-4}$ (Figure 2d). At lower protein concentration the NT-SUP-CT chains seem to preferentially precipitate at the oil droplets surface. Increasing the content of protein five times (Figure $2 \mathrm{c}$ ) resulted in the formation of protein aggregates together with nanocapsules with a $\bar{D}_{Z T E M}$ around 185 (STD of 1.35) nm and $\bar{D}_{Z D L S}$ around $250 \mathrm{~nm}$ (PDI of 0.20) (Table 3). At this critical concentration, all oil droplets are encapsulated, and some protein leftovers aggregate together forming the observed darks spots on the TEM image. Further doubling the protein content (Figure $2 \mathrm{~d}$ ) induced the formation of essentially large aggregates.

Nanocapsules were conveniently cross-linked using a $6.5 \times 10^{-6}$ mass fraction of IPDI. A lower content of IPDI was tested in a trial to optimize the amount of cross-linker used. Figure 2 compares two samples prepared at an acetone content of $30 \mathrm{wt} \%$, a protein mass fraction of $10^{-5}$ and an oil mass fraction of $10^{-4}$ cross-linked with $6.5 \times 10^{-6}$ wt.\% (Figure 2b) and $1.6 \times 10^{-6}$ (Figure 2e). Even though the size and dispersity measured from the dried nanocapsules (TEM) did not change, a higher average diameter size measured by DLS $(300 \mathrm{~nm})$ indicates that a lower content of IPDI results in swollen objects and therefore a lower crosslinking density (Table 3). Besides, the background of the TEM images of the samples containing less IPDI appears contaminated, suggesting incomplete crosslinking of the proteins shells and precipitation of the free protein chains upon solvent drying. Most importantly, crosslinking of the protein shell with adequate amounts of IPDI (Table 3b) ensures efficient stabilization of protein-based nanocapsules in aqueous conditions. TEM characterization confirmed that the morphology of the nanocapsules was maintained after evaporation of acetone (Figure S6a). Accordingly, DLS analyses underlined the presence of a main population corresponding to individual nanocapsules (main peak) and a minor population over the $\mu \mathrm{m}$ revealing a little tendency of these objects to aggregate in aqueous solutions (Figure S6b).

The secondary structure of the suckerin-silk chains incorporated in the nanocapsules' shells was further studied by FTIR. Multiple peaks can be assigned to a type of secondary structure, as there may be multiple segments of proteins with the same structural class, thus exhibiting absorption at multiple frequencies (add ref:Byler et al). We have compared amide I bands on the FTIR spectra of the nanocapsule sample after acetone evaporation (Figure $2 \mathrm{~g}$ ) and of the suckerin-silk dissolved in aqueous buffer at a concentration of $0.1 \mathrm{mg} / \mathrm{ml}$ (Figure 2f). 

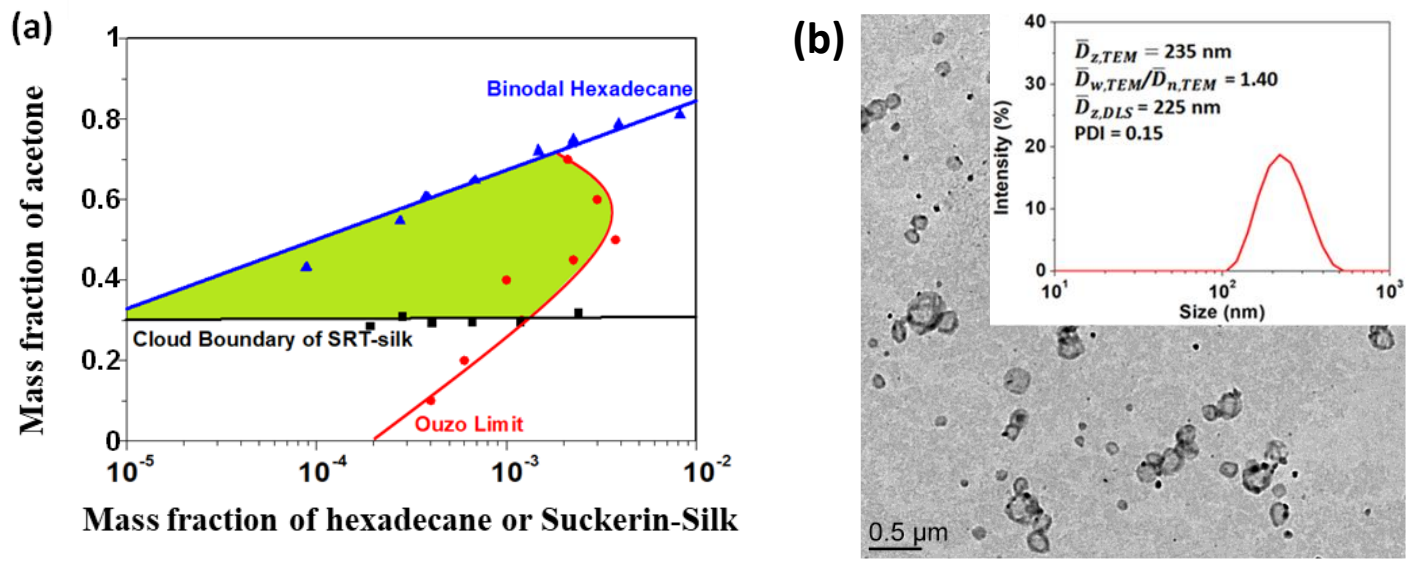

Figure 3. Hexadecane droplets encapsulated in NT-SUP-CT shells. (a) Phase diagram of the system acetone/water/hexadecane and cloud boundary of NT-SUP-CT. Domain of coprecipitation stained in green. (b) TEM image and DLS spectrum of nanocapsules prepared at $30 / 70$ acetone/aqueous buffer ratio, $10^{-5}$ mass fraction of NT-SUP-CT, $10^{-4}$ mass fraction of hexadecane and $6.5 \times 10^{-6}$ mass fraction of IPDI.

When the protein was self-assembled into nanocapsule shells, a clear increase in $\beta$-sheet content could be deduced from the increase of the characteristic $\beta$-sheet band between 1624 to $1641 \mathrm{~nm} .{ }^{31}$ Tests were also performed to investigate the possibility of stabilizing the NT-SUP-CT membranes by triggering the temperature sensitive $\alpha$ coil - $\beta$-sheet transition. A sample prepared with an acetone content of $30 \mathrm{wt} \%$, an oil mass fraction of $10^{-4}$ and a protein mass fraction of $10^{-5}$ was heated in a closed vial to $50{ }^{\circ} \mathrm{C}$ for $30 \mathrm{~min}$. Unfortunately, this strategy did not seem to successfully freeze the nanocapsules structure, since large protein aggregates were observed by TEM (Figure S8).

Finally, to demonstrate that our approach is not limited to one specific oil and can be generalized to a range of hydrophobic solutes, we further intended to entrap hexadecane nanodroplets into suckerin-silk shells. The hexadecane/acetone/water phase diagram has been previously used by Yan et al. to synthesize glyconanocapsules (Figure 3a). ${ }^{10}$ Based on our previous investigations with miglyol, we proceeded to the solvent shifting targeting a final acetone content of $30 \mathrm{wt} \%, 10^{-5}$ mass fraction of suckerin-silk, $10^{-4}$ mass fraction of hexadecane and $6.5 \times 10^{-6}$ mass fraction of IPDI. Similar to the nanoprecipitation system involving miglyol as hydrophobic solute, well-defined oil-filled proteinaceous nanocapsules with an average diameter around $230 \mathrm{~nm}$ were conceived in one step (Figures $3 \mathrm{~b}$ ).

In conclusion, the new designed fusion protein suckerin with soluble silk tags exhibits an enhanced solubility in aqueous buffer at a $\mathrm{pH}$ (7.4), making it compatible with the nanoprecipitation technique and a good candidate for generating oil-filled protein nanocapsules. In addition, a clear change in solution turbidity can be observed when the aqueous solution of protein is titrated with acetone, which allows the identification of the cloud point boundary and therefore the determination of the domain of composition where oil filled nanocapsules can be generated. IPDI-driven crosslinking was efficient at stabilizing the suckerin-silk shells around the oily cores, with higher concentration of the cross-linker reducing swelling of the shells in the hydrated state. Suckerin-silk-shelled nanocapsules with a mean diameter around $200 \mathrm{~nm}$ were fabricated using miglyol or hexadecane as oils. These results validating that NT-SUP-CT fusion proteins show potential to be used in combination with a variety of hydrophobic therapeutics to design proteinaceous nanocapsules by nanoprecipitation form a strong basis for a broad area of applications relying on encapsulation technologies.

\section{ASSOCIATED CONTENT}

\section{Supporting Information.}

Experimental section detailing the expression and purification of the fusion protein. Nanocapsule fabrication protocol. DLS of the suckerin-silk protein at different pHs. DLS spectrum of the suckerin-19 protein at $\mathrm{pH} 10$. Suckerin-19 nanocapsule TEM image. TEM images of failed samples of suckerin-silk nanocapsules. Second derivatives of FTIR spectra. Full imgaes of TEM photos given in the main text. This material is available free of charge via the Internet at http://pubs.acs.org.

\section{AUTHOR INFORMATION}

\section{Corresponding Authors}

Julien Bernard - Univ Lyon, INSA Lyon, CNRS, IMP UMR 5223, F-69622, Villeurbanne, France

Email: julien.bernard@insa-lyon.fr

Ali Miserez - Center for Sustainable Materials, School of Materials Science and Engineering, Nanyang Technological University (NTU), 50 Nanyang Drive, 637553 Singapore, and School of Biological Sciences, NTU, 60 Nanyang Drive, 637551 Singapore. Email: ali.miserez@ntu.edu.sg

\section{Authors}

Ricardo Ramos - Univ Lyon, INSA Lyon, CNRS, IMP UMR 5223, F-69622, Villeurbanne, France

Bartosz Gabryelczyk - Center for Sustainable Materials (SusMat), School of Materials Science and Engineering, Nanyang Technological University (NTU), 50 Nanyang Drive, 637553 Singapore

Luxiao Chai - Univ Lyon, INSA Lyon, CNRS, IMP UMR 5223, F69622, Villeurbanne, France

Deepankumar Kanagavel - SusMat, School of Materials Science and Engineering, NTU, 50 Nanyang Drive, 637553 Singapore Xibo Yan - Univ Lyon, INSA Lyon, CNRS, IMP UMR 5223, F69622, Villeurbanne, France

Kenrick Koh - SusMat, School of Materials Science and Engineering, NTU, 50 Nanyang Drive, 637553 Singapore, and NTU Institute for Health Technologies, Interdisciplinary Graduate Programme, NTU, 637335 Singapore

François Ganachaud - Univ Lyon, INSA Lyon, CNRS, IMP UMR 5223, F-69622, Villeurbanne, France

\section{Present Addresses}

Bartosz Gabryelczyk - Department of Bioproducts and Biosystems, School of Chemical Engineering, Aalto University, Kemistintie 1, 02150 Espoo, Finland.

\section{Author Contributions}

The manuscript was written through contributions of all authors. All authors have given approval to the final version of the manuscript. 


\section{Funding Sources}

This research was supported by a joint "French Agency for National Research (ANR)"/"Singapore National Research Foundation (NRF)" grant awarded to J.B (PREPROPOSAL, ANR-15-CE09-0021) and A.M. (NRF2015-NRF-ANR000). A.M. also acknowledges financial support from the Singapore Ministry of Education (MOE) through a Tier 2 grant (MOE 2018-T2-1-043).

\section{ACKNOWLEDGMENT}

Dr L. C. acknowledges CSC for a $\mathrm{PhD}$ grant. The authors acknowledge Pierre Alcouffe and the Centre Technologique des Microstructures (CT $\mu$, Université Lyon 1) for their help with microscopy analyses and Shu Hui Hiew for her help with FTIR experiments.

\section{REFERENCES}

(1) V. Wagner, A. Dullaart, A.-K. Bock, A. Zweck, The emerging nanomedicine landscape. Nat. Biotechnol. 2006, 24, 1211.

(2) O. C. Farokhzad, R. Langer. Impact of nanotechnology on drug delivery. ACS Nano 2009, 3, 1, 16.

(3) N. M. Molino, S.-W. Wang. Caged protein nanoparticles for drug delivery. Curr. Opin. Biotechnol. 2014, 28, 75.

(4) E. A. Berckman, E. J. Hartzell, A. A. Mitkas, Q. Sun, W. Chen. Biological Assembly of Modular Protein Building Blocks as Sensing, Delivery, and Therapeutic Agents. Annu. Rev. Chem. Biomol. Eng. 2020, 11, 35 .

(5) M. S. Ardejani, X. L. Chok, C. J. Foo, B. P. Orner. Complete shift of ferritin oligomerization toward nanocageassemblyvia engineered protein-protein interactions. Chem. Commun. 2013, 49, 3528.

(6) Y. Yang, Y. Jia, L. Gao, J. Fei, L. Dai, J. Zhao, J. Li. Fabrication of autofluorescent protein coated mesoporous silicananoparticles for biological application. Chem. Commun. 2011, 47, 12167.

(7) Y. Lv, F. Yang, X. Li, X. Zhang, S. Abbas. Formation of heat-resistan nanocapsules of jasmine essential oil via gelatin/gum arabic based complex coacervation. Food Hydrocoll. 2014, 35, 305.

(8) M. Fichter, K. Piradashvili, A. Pietrzak-Nguyen, L. Pretsch, G. Kuhn, S. Strand, M. Knuf, F. Zepp, F. R. Wurm, V. Mailänder, K. Landfester, S. Gehring. Polymeric hepatitis $\mathrm{C}$ virus non-structural protein $5 \mathrm{~A}$ nanocapsules induce intrahepatic antigen-specific immune responses. $\mathrm{Bi}$ omaterials 2016, 108, 1.

(9) K. Piradashvili, J. Simon, D. Paßlick, J. R. Höhner, V. Mailänder, F R. Wurm, K. Landfester. Fully degradable protein nanocarriers by orthogonal photoclick tetrazole-ene chemistry for the encapsulation and release. Nanoscale Horiz. 2017, 2, 297.

(10) X. Yan, M. Delgado, A. Fu, P. Alcouffe, S. G. Gouin, E. Fleury, J L. Katz, F. Ganachaud, J. Bernard. Simple but precise engineering of functional nanocapsules through nanoprecipitation. Angew. Chem. Int Ed. 2014, 53, 6910.

(11) X. Yan, R. Ramos, E. Hoibian, C. Soulage, P. Alcouffe, F. Ganachaud, J. Bernard. Nanoprecipitation of PHPMA (Co)Polymers into nanocapsules displaying tunable compositions, dimensions, and surface properties. ACS Macro Lett. 2017, 6, 447.

(12) X. Yan, P. Alcouffe, G. Sudre, L. David, J. Bernard, F. Ganachaud. Modular construction of single-component polymer nanocapsules through a one-step surfactant-free microemulsion templated synthesis. Chem. Commun. 2017, 53, 1401.

(13) X. Yan, R. A. N. S. Ramos, P. Alcouffe, L. E. Munoz, R. O. Bilyy, F. Ganachaud, J. Bernard. Programmable hierarchical construction of mixed/multilayered polysaccharide nanocapsules through simultaneous/sequential nanoprecipitation steps. Biomacromolecules 2019, 20, 3915.

(14) Y. Ping, D. Ding, R. A. N. S. Ramos, H. Mohanram, K. Deepankumar, J. Gao, G. Tang, A. Miserez. Supramolecular $\beta$-sheets stabilized protein nanocarriers for drug delivery and gene transfection. ACS Nano 2017, 11, 4528.
(15) D. Ding, J. Pan, S. H. Lim, S. Amini, L. Kang, A. Miserez. Squid suckerin microneedle arrays for tunable drug release. J. Mater. Chem. B 2017, 5, 8467.

(16) A. Miserez, J. C. Weaver, P. B. Pedersen, T. Schneeberk, R. T. Hanlon, D. Kisailus, H. Birkedal. Microstructural and biochemical characterization of the nanoporous sucker rings from dosidicus gigas. Adv. Mater. 2009, 21, 401.

(17) P. A. Guerette, S. Hoon, Y. Seow, M. Raida, A. Masic, F. T. Wong, V. H. B. Ho, K. W. Kong, M. C. Demirel, A. Pena-Francesch, S. Amini, G. Z. Tay, D. Ding, A. Miserez. Accelerating the design of biomimetic materials by integrating RNA-seq with proteomics and materials science. Nat. Biotechnol. 2013, 31, 908.

(18) S. H. Hiew, A. Miserez. Squid sucker ring teeth: multiscale structure-property relationships, sequencing, and protein engineering of a thermoplastic biopolymer. ACS Biomater. Sci. Eng. 2017, 3, 680.

(19) P. A. Guerette, S. Hoon, D. Ding, S. Amini, A. Masic, V. Ravi, B. Venkatesh, J. C. Weaver, A. Miserez. Nanoconfined $\beta$ sheets mechanically reinforce the supra-biomolecular network of robust squid sucker ring teeth. ACS Nano 2014, 8, 7170.

(20) A. Kumar, H. Mohanram, K. W. Kong, R. Goh, S. Hoon, J. Lescar, A. Miserez. Supramolecular propensity of suckerin proteins is driven by $\beta$-sheets and aromatic interactions as revealed by solution NMR. Biomater. Sci. 2018, 6, 2440.

(21) V. Latza, P. A. Guerette, D. Ding, S. Amini, A. Kumar, I. Schmidt, S. Keating, N. Oxman, J. C. Weaver, P. Fratzl, A. Miserez, A. Masic. Multi-scale thermal stability of a hard thermoplastic protein-based material. Nat. Commun. 2015, 6.

(22) D. Ding, P. A. Guerette, J. Fu, L. Zhang, S. A. Irvine, A. Miserez. From soft self-healing gels to stiff films in suckerin-based materials through modulation of crosslink density and $\beta$-sheet content. Adv. Mater. 2015, 27, 3953.

(23) D. Ding, P. A. Guerette, S. Hoon, K. W. Kong, T. Cornvik, M. Nilsson, A. Kumar, J. Lescar, A. Miserez. Biomimetic production of silk-like recombinant squid sucker ring teeth proteins. Biomacromolecules 2014, 15,3278 .

(24) X. Yan, M. Delgado, J. Aubry, O. Gribelin, A. Stocco, F. BoissonDa Cruz, J. Bernard, F. Ganachaud. Central role of bicarbonate anions in charging water/hydrophobic interfaces. J. Phys. Chem. Lett. 2018, 9, 96 (25) M. Andersson, Q. Jia, A. Abella, X.-Y. Lee, M. Landreh, P. Purhonen, H. Hebert, M. Tenje, C. V. Robinson, Q. Meng, G. R. Plaza, J. Johansson, A. Rising. Biomimetic spinning of artificial spider silk from a chimeric minispidroin. Nat. Chem. Biol. 2017, 13, 262.

(26) F. Hagn, L. Eisoldt, J. G. Hardy, C. Vendrely, M. Coles, T. Scheibel, H. Kessler. A conserved spider silk domain acts as a molecular switch that controls fibre assembly. Nature 2010, 465, 239.

(27) G. Askarieh, M. Hedhammar, K. Nordling, A. Saenz, C. Casals, A. Rising, J. Johansson, S. D. Knight. Self-assembly of spider silk proteins is controlled by a pH-sensitive relay. Nature 2010, 465, 236.

(28) N. Kronqvist, M. Sarr, A. Lindqvist, K. Nordling, M. Otikovs, L. Venturi, B. Pioselli, P. Purhonen, M. Landreh, H. Biverstål, Z. Toleikis, L. Sjöberg, C. V. Robinson, N. Pelizzi, H. Jörnvall, H. Hebert, K. Jaudzems, T. Curstedt, A. Rising, J. Johansson. Efficient protein production inspired by how spiders make silk. Nat. Commun. 2017, 8, 15504.

(29) M. Sarr, N. Kronqvist, G. Chen, R. Aleksis, P. Purhonen, H. Hebert, K. Jaudzems, A. Rising, J. Johansson. A spidroin-derived solubility tag enables controlled aggregation of a designed amyloid protein. FEBS J. $2018,285,1873$.

(30) S. H. Hiew, A. Sánchez-Ferrer, S. Amini, F. Zhou, J. Adamcik, P. Guerette, H. Su, R. Mezzenga, A. Miserez. Squid Suckerin Biomimetic Peptides Form Amyloid-like Crystals with Robust Mechanical Properties. Biomacromolecules 2017, 18, 4240.

(31) A. Barth. Infrared spectroscopy of proteins. Biochimica et biophysica Acta (BBA), 2007, 1767, 1073. 
Insert Table of Contents artwork here

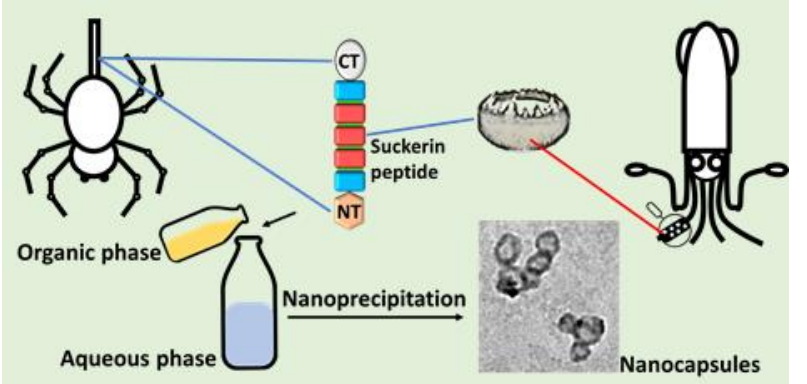

